\title{
The long and short of residual force enhancement non-responders
}

\author{
Geoffrey A. Power ${ }^{1} \cdot$ Avery Hinks $^{1} \cdot$ Parastoo Mashouri ${ }^{1} \cdot$ Vincenzo S. Contento $^{1} \cdot$ Jackey Chen $^{1}$
}

Published online: 13 October 2020

(c) Springer-Verlag GmbH Germany, part of Springer Nature 2020

Residual force enhancement ( $\mathrm{rFE}$ ) refers to the 'extra' force that is achieved in an isometric contraction following active muscle lengthening as compared to a strictly isometric contraction (i.e., fixed end contraction) performed at the same muscle length and level of neuromuscular activation. As an intrinsic property of muscle contractility, $\mathrm{rFE}$ is present from the single sarcomere, to whole muscles of humans during electrical stimulation and voluntary activation (Chapman et al. 2018; Seiberl et al. 2015).

In the current issue of EJAP, Bakenecker et al. (2020) investigated the effect of muscle-tendon unit length on transient force enhancement ( $\mathrm{tFE}$; force production during an eccentric contraction) and $\mathrm{rFE}$ (steady-state isometric force following active lengthening). They found that $\mathrm{tFE}$ was indeed present at short and long muscle-tendon unit lengths, while rFE was blunted at shorter lengths. This finding is consistent with the phenomenological mechanisms of $\mathrm{rFE}$, which shows greater magnitudes of $\mathrm{rFE}$ on the descending limb of the length-tension relationship as compared with the plateau or ascending limb (Rassier and Herzog 2004). However, it is possible that significant rFE was not observed at short muscle lengths, because $3-4$ of the 12 participants displayed no rFE. Conversely, all participants showed rFE at long muscle lengths (see Fig. 3). This offers an important discussion point, from reduced muscle preparations we would expect rFE across all operating muscle lengths, just less at shorter than at longer lengths (Rassier and Herzog 2004). It does not necessarily need to be stated that humans performing voluntary contractions are not isolated muscles in a bath of chemicals, thus a myriad

Electronic supplementary material The online version of this article (https://doi.org/10.1007/s00421-020-04511-5) contains supplementary material, which is available to authorized users.

Geoffrey A. Power

gapower@uoguelph.ca

1 Neuromechanical Performance Research Laboratory, Department of Human Health and Nutritional Sciences, College of Biological Sciences, University of Guelph, Guelph, ON N1G 2W1, Canada of neuromuscular factors could contribute to blunting rFE. With this, an important consideration in the rFE field is the phenomenon of non-responders.

Non-responders have been identified as human research participants exhibiting negligible or no rFE. Various factors related to neural excitation/inhibition and motor unit recruitment, as well as mechanical factors such as series compliance could all contribute to the variability in reported magnitudes of rFE. Understanding the underlying mechanisms of muscle contractility is crucial, but the implications of an intact human neuromuscular system on modulating rFE cannot be overlooked when investigating this historydependent property of muscle. Below we discuss some of these considerations.

Level of activation and fibre type: Based on results from the only study directly investigating the EMG-force relationship following active lengthening, relative values (i.e., steady-state force following active lengthening minus isometric force, divided by isometric force) of $\mathrm{rFE}(\sim 5 \%)$ for the human dorsiflexors appear to be similar for tibialis anterior activation levels between 20 and 100\% MVC (Paquin and Power 2018). Meanwhile, Oskouei and Herzog (2005) determined that increasing levels of activation $(10 \%, 30 \%$, and $60 \% \mathrm{MVC}$ ) led to greater absolute values of $\mathrm{rFE}$, as well as more responders (i.e., $4 / 12$ subjects exhibiting $\mathrm{rFE}$ at $10 \%$ MVC vs. 10/12 at 60\% MVC), for the human adductor pollicis. Therefore, the level of neuromuscular activation appears to affect absolute, but not relative, values of rFE. One way to get around this apparent activation dependency is with electrically evoked contractions. Lee and Herzog (2002) performed voluntary and electrically-evoked contractions of the adductor pollicis muscle and found no difference in $\mathrm{rFE}$ following slow speed lengthening contractions (16\% vs. $17 \%$, respectively), however, during the faster speed lengthening contractions, voluntary $\mathrm{rFE}$ was less than electrically stimulated (12\% vs. $17 \%$, respectively) — thus, it seems with increasing lengthening speeds, there may be factors affecting voluntary performance, such as neural inhibition.

To investigate whether resistance training may alter rFE, Chen and Power (2019) performed a 4-week study 
biased to concentric and eccentric training. They found that concentric training increased $\mathrm{rFE}$ while eccentric training decreased $\mathrm{rFE}$ for the human ankle dorsiflexors. The rFE increase was attributed to an increase in the number of responders (from 11/15 to 15/15) following training, while the rFE decrease was attributed to increased antagonist muscle (soleus) co-activation. Given that training only lasted 4 weeks-during which adaptations are predominantly neural-these results point to a potential neural contribution to non-responders. More carefully controlled longitudinal studies and day-to-day reliability measures of non-responders are warranted to fully understand the phenomenon of non-responders.

If muscle fibre type contributes to the magnitude of $\mathrm{rFE}$, then recruiting high threshold motor units would presumably increase responders and explain the activation dependency often observed. The first study investigating muscle fibre type differences in $\mathrm{rFE}$ was performed by Ramsey et al. (2010), which compared rFE in the soleus (SOL) and extensor digitorum longus (EDL) of rats. Ramsey et al. (2010) reported $\sim 55 \%$ greater $\mathrm{rFE}$ values for the $\mathrm{EDL}$, which is comprised of primarily (95-100\%) type II fibres, compared to the SOL, which is primarily (75-80\%) type I fibres. The authors speculate that the expression of shorter, stiffer titin isoforms-which contribute greater passive force to total force production-in predominantly fast-twitch muscles may contribute to the fibre type differences in rFE. In contrast to prior studies, Pinnell et al. (2019) found no fibre type differences for $\mathrm{rFE}$ in human single fibres of the vastus lateralis (VL), and suggested this may be due to fewer fibre type differences in titin isoforms within a single muscle compared to across different muscles. In humans at least, differences in fibre type across muscles may not be a determining factor in the incidence of non-responders.

Central nervous system excitability: Reduced central drive, increased inhibition, or a combination of both could contribute to the incidence of non-responders. While a reduction in agonist activation is almost certain, it appears to be related, in part, to spinal excitability (Sypkes et al. 2018). Contento et al. (2019) reported an increased tendonevoked inhibitory reflex during the rFE state compared to strictly isometric contractions. These results likely indicate inhibitory feedback onto the agonist motoneuron pool that is arising from a tension-dependent source within the tendon, most likely the golgi tendon organ, and subsequently reducing spinal excitability (Sypkes et al. 2018). Surprisingly, when vibration was applied to the muscle, vibratory excitation of muscle spindles does not appear to alter rFE (Dalton et al. 2018). Thus, if certain individuals experience more tension-dependent inhibition during and/or following active lengthening, this alteration in descending drive or spinal excitability would likely contribute to the non-responder phenomenon.
Muscle-tendon unit compliance: In some cases, more rFE may just be less residual force depression (rFD). When Raiteri and Hahn (2019) increased the stiffness of the human tibialis anterior muscle-tendon unit (MTU) at the onset of a 'fixed-end' (i.e., isometric) contraction via a small, quick joint rotation, they found that isometric force was increased, owing to reduced internal shortening of fascicles upon activation. In other words, even for isometric contractions where no joint angle changes occur, MTU compliance appears to play a role in $\mathrm{rFD}$, whereby a more compliant tendon permits greater fascicle shortening, and thus, more absolute shortening-induced $\mathrm{rFD}$ (and vice versa). Moreover, for 'fixedend' isometric contractions, where internal shortening of fascicles can occur, decreasing levels of activation result in lower absolute amplitudes of shortening-induced rFD, owing to a smaller magnitude of internal shortening and a potential triggering of rFE-related mechanisms as force drops and fascicles actively relax/lengthen (Raiteri and Hahn 2019). In short, for conventional rFE contractions that compare back to a reference isometric contraction, the level of activation likely influences absolute values of rFE. However, if isometric contractions were adjusted for any potential internal shortening of fascicles, it might reveal underestimations of rFD and possible overestimations of rFE in the literature. What we may see is not really force enhancement but less force depression in the stretched muscle compared to the isometric reference conditions.

How do we deal with non-responders in a data set? To properly answer this question, there needs to be a better understanding of why non-responders exist. Once again, tracking the dynamic behaviour of muscle architecture during contractions might be a good place to start, and this was an elegant aspect of the study by Bakenecker et al. (2020). Depending on the research question, to limit non-responders in a data set, the available literature would point to using large joint excursions biased to long muscle-tendon unit lengths, with high levels of activation. Although, a nonresponder in and of themselves may provide critical insight and clues into the everyday relevance of $\mathrm{rFE}$. Therefore, it is important to consider that while these intrinsic contractile properties of muscle always exist during carefully controlled in vitro experiments, the complex human neuromuscular system may or may not always follow suit.

Author contributions All authors contributed to the writing, editing, and approved the submitted version.

\section{Compliance with ethical standards}

Conflict of interest No conflicts of interest, competing interest of any sort, financial or otherwise, are declared by the authors. 


\section{References}

Bakenecker P, Raiteri BJ, Hahn D (2020) Force enhancement in the human vastus laterals is muscle-length dependent following stretch but not during stretch. Eur J Appl, Physiol (In Press)

Chapman N, Whitting J, Broadbent S, Crowley-McHattan Z, Meir R (2018) Residual force enhancement in humans: a systematic review. J Appl Biomech 34:240-248

Chen J, Power GA (2019) Modifiability of the history dependence of force through eccentric and concentric biased resistance training. J Appl Physiol 126:647-657

Contento VS, Dalton BH, Power GA (2019) The inhibitory tendonevoked reflex is increased in the torque-enhanced state following active lengthening compared to a purely isometric contraction. Brain Sci 10(1): 13

Dalton BH, Contento VS, Power GA (2018) Residual force enhancement during submaximal and maximal effort contractions of the plantar flexors across knee angle. J Biomech 78:70-76

Lee HD, Herzog W (2002) Force enhancement following muscle stretch of electrically stimulated and voluntarily activated human adductor pollicis. J Physiol 545:321-330

Oskouei AE, Herzog W (2005) Observations on force enhancement in submaximal voluntary contractions of human adductor pollicis muscle. J Appl Physiol 98:2087-2095

Paquin J, Power GA (2018) The history dependence of the EMG-force relationship during ankle dorsiflexion. J Electromyogr Kinesiol 41:109-115
Pinnell RAM, Mashouri P, Mazara N, Weersink E, Brown SHM, Power GA (2019) Residual force enhancement and force depression in human single muscle fibres. J Biomech 91:164-169

Raiteri B, Hahn D (2019) A reduction in compliance or activation level reduces residual force depression in human tibialis anterior. Act Physiol (Oxf) 225:e13198

Ramsey KA, Bakker AJ, Pinniger GJ (2010) Fiber-type dependence of stretch-induced force enhancement in rat skeletal muscle. Muscle Nerve 42:769-777

Rassier DE, Herzog W (2004) Considerations on the history dependence of muscle contraction. J Appl Physiol 96:419-427

Seiberl W, Power GA, Hahn D (2015) Residual force enhancement in humans: current evidence and unresolved issues. J Electromyogr Kinesiol 25:571-580

Sypkes CT, Kozlowski BJ, Grant J, Bent LR, McNeil CJ, Power GA (2018) The influence of residual force enhancement on spinal and supraspinal excitability. PeerJ 6:e5421

Publisher's Note Springer Nature remains neutral with regard to jurisdictional claims in published maps and institutional affiliations. 